\title{
The importance of intracytoplasmic DPAS positivity in fine needle aspirates of breast lesions
}

\author{
S J Johnson, V Wadehra
}

\begin{abstract}
Aims-For many years the presence of strong intracytoplasmic periodic acid Schiff (PAS) positive, diastase resistant (DPAS) staining within atypical cells has been used in this laboratory as a marker for carcinoma in fine needle aspirates from breast lesions. The aim of the current study was to document such DPAS positivity across the spectrum from benign to malignant breast disease and assess its value in the upgrading of cytology reports.
\end{abstract}

Methods-Over a six month period, 315 aspirates were studied, each having sufficient cellular material for DPAS staining to be carried out on one whole slide. DPAS staining was recorded semiquantitatively as negative, equivocal, or positive $(+,++$, or +++$)$. The cytology results were correlated with any subsequent histology performed on these patients.

Results-DPAS positive material was seen in both intracellular and extracellular locations. Care was needed in its interpretation. Occasional cells with apparently genuine intracytoplasmic positivity $(+)$ were found in both benign and malignant cases. Frequent or particularly strong intracellular DPAS positivity $(++$, $+++)$ correlated best with malignancy. Two cases were reliably upgraded from immediate reports suspicious of malignancy to final reports diagnostic of malignancy on the basis of the intracytoplasmic DPAS staining.

Conclusion-Strict criteria are required for the interpretation of intracytoplasmic DPAS positivity and routine cytological appearances should also be taken into account. Weak positivity in occasional cells, especially in flat epithelial sheets, may be seen in benign lesions and is not reliable as a marker of malignancy. DPAS positivity with internal structure and producing nuclear indentation, especially in dissociated or atypical cells, correlates well with malignant histology and can be reassuring in the cytological diagnosis of malignancy.

(f Clin Pathol 2001;54:146-151)

Keywords: fine needle aspiration of breast; mucin positivity; periodic acid Schiff positive, diastase resistant cells

Fine needle aspiration (FNA) is a fast, accurate, and minimally invasive means of obtaining a preoperative cytological diagnosis in symptomatic and screen detected breast lesions. It is essential to ensure high accuracy of FNA results with respect to subsequent histological diagnosis to maintain surgical and radiological confidence in the service.

The department of cytology at the Royal Victoria Infirmary, Newcastle upon Tyne, UK receives approximately 1900 breast FNA specimens each year, one third of which derive from the local Breast Screening and Assessment Centre. The FNAs are taken by radiologists and surgeons, including trainees. Most of the specimens are from dedicated one stop breast clinics; the cytopathologist issues an immediate verbal report to the clinician during the clinic based on staining of one or two direct smears for each needle pass. The final written cytology report follows staining of any remaining slides and the needle washout preparations. ${ }^{1}$ The consultant rota is arranged so that, whenever possible, the immediate and final reports are issued by different cytopathologists to serve as an internal quality control.

We have noted that FNAs from breast carcinoma often contain intracytoplasmic material positive for periodic acid Schiff after diastase (DPAS). For many years we have used such DPAS staining to assist in making a reliable final diagnosis of malignancy in difficult breast FNA cytology samples that are otherwise suspicious of malignancy.

The aim of our current study was to assess DPAS staining in breast FNAs across the entire spectrum from benign to malignant breast disease, and to audit prospectively the influence of any staining on the final report.

\section{Methods}

Our study was performed over a six month period from September 1998 to April 1999. FNA samples were included only when there were sufficient cells on more than one slide to enable the use of an entire slide for DPAS staining; this gave 315 aspirates for study out of a total of 1149 breast FNAs received during that period.

STAINING FOR IMMEDIATE AND FINAL REPORTING For the immediate report, the one or two most cellular spread slides from each needle pass were stained by hand with a Diff-Quik (DQ) stain. For final reporting, any remaining spread slides were stained mechanically with MGG (May-Grünwald-Giemsa). A cytospin preparation was made from the needle washout fluid (when received) and was stained mechanically with a Papanicolau (Pap) stain. Staining for final reporting usually took place on the day of the immediate report for morning clinics or early the next day for afternoon clinics. 
DPAS STAINING METHOD

DPAS staining was performed on either an unstained slide, if available, or a destained MGG or DQ slide after cytological assessment. The slide was covered with damp filter paper, fresh saliva was applied to the filter paper, and the slide was incubated for 30 minutes at $37^{\circ} \mathrm{C}$. The slide was then rinsed in water, covered with $1 \%$ periodic acid (BDH-Merck Ltd, Lutterworth, UK) for eight minutes, rinsed in distilled water, covered with Schiff's reagent (BDH-Merck Ltd) for 30 minutes, washed in running water for 30 minutes, and counterstained with haematoxylin.

ASSESSMENT OF DPAS STAINED SLIDES FOR

ADEQUACY OF STAINING

It is essential that the DPAS staining is strong. Starch granules, present as a contaminant in most slides, provide a useful internal positive control. In a correctly stained slide these should be intensely and uniformly magenta in colour from the edge to the centre; central pallor indicates weaker staining and can lead to false negative staining in the cells themselves. Any weakly stained slides were restained without prior destaining.

\section{REPORTING OF THE CASES}

All the cases were reported using our usual cytological criteria. The national $\mathrm{C} 1-5$ grading has been recorded in this department since 1979 as an AC (aspiration cytology) grade 0 to $5{ }^{1} \mathrm{AC} 0$ is an inadequate specimen containing no breast duct epithelial cells or just one group; $\mathrm{AC} 1$ is also inadequate, containing less than six groups of epithelial cells; AC2 is an adequate sample containing at least six groups (at least 12 duct epithelial cells in each group) of benign cells (with an additional diagnosis suggested when appropriate-for example, fibrocystic change or fibroadenoma); AC3 is a sample with atypia that is probably benign; AC4 reflects atypia that is probably malignant (suspicious of carcinoma); and AC5 is diagnostic of carcinoma (with a type and grade given whenever possible-for example, ductal, lobular, mucinous). We subdivide AC3 specimens into two groups: those with only minor atypia and those with more worrying atypia in which advice is given on excising the lesion for a histological diagnosis.

For intracellular DPAS positivity to be considered relevant we required staining that had a definite magenta colour, was fully within the cytoplasm, and was round in shape, with a well defined crisp edge. Such intracellular DPAS staining was recorded semiquantitatively, as follows: negative; \pm , equivocal staining (taken as negative when assessing results); +,

Table 1 Aspiration cytology (AC) grades and subsequent histology results

\begin{tabular}{lllll}
\hline $\begin{array}{l}\text { AC grade as finally } \\
\text { reported }\end{array}$ & Number of cases & $\begin{array}{l}\text { No histology } \\
\text { performed }\end{array}$ & $\begin{array}{l}\text { Benign on } \\
\text { histology }\end{array}$ & $\begin{array}{l}\text { Malignant on } \\
\text { histology }\end{array}$ \\
\hline AC2 & 137 & 126 & 10 & 1 \\
AC3 minor & 50 & 41 & 7 & 2 \\
AC3 excise & 26 & 3 & 18 & 5 \\
AC4 & 47 & 3 & 14 & 30 \\
AC5 & 155 & 44 & 1 & 110 \\
Total & 315 & 217 & 50 & 148 \\
\hline
\end{tabular}

occasional cells with definite staining; ++ , an intermediate number of cells with definite staining; +++, numerous positive cells or particularly strong staining. Note was taken of any other intracellular or extracellular staining. Any influence of the DPAS result on the final issued report was documented. All cases were checked for subsequent histology results (core biopsy, open or excision biopsy, or mastectomy) from the same lesion as the FNA sampling.

Positive predictive values (PPVs) for malignant histology were calculated as: (true positives (malignant histology))/(true positives + false positives (benign histology) $\times 100 \%$.

\section{Results}

\section{ROUTINE CYTOLOGY}

Of the 315 FNAs, 137 were reported as benign, 50 as minor atypia, 26 as atypia with excision advised, 47 as suspicious of malignancy, and 155 as diagnostic of malignancy. Many of the AC4 and AC5 aspirates contained cells with intracytoplasmic lumina or cytoplasmic vacuoles evident on DQ/MGG or Pap stains. The intracytoplasmic lumina were usually single and smaller than the nucleus, with a thick wall. Some contained a central darkly staining dot, creating the "magenta bodies" so characteristic of aspirates from a breast carcinoma. In a few cells, the vacuoles or lumina produced a signet ring cell morphology with compression of the nucleus to a crescent shape. In many of the sheets of benign epithelial cells (AC2 and AC3 cases) there were occasional cells with small intracytoplasmic secretory vacuoles. Cells from all types of aspirate showed scattered small clear spaces resembling artefactual bubbles in the staining.

CORRELATION OF FNA AC GRADE WITH SUBSEQUENT HISTOLOGY Histology was performed after 198 of the FNAs (table 1). For most of the AC2 and AC3 minor atypia FNAs there was no histology (clinical and radiological decision), but when performed it was benign in most cases. In one symptomatic patient with a clinically malignant lump the initial FNA was reported as AC2 and a repeat FNA as AC3 excise (DPAS negative); the initial core biopsy was negative but an excision specimen showed carcinoma. The FNAs and core biopsy in this case presumably represent sampling errors. One AC3 minor atypia from an area of microcalcification was accompanied by an inconclusive core biopsy (no calcification) but ductal carcinoma in situ (DCIS) was present on the excision biopsy; again this presumably reflects sampling error for the FNA and core. Another AC3 minor atypia case was followed by malignant histology, and proved to be a grade 1 invasive ductal carcinoma.

For AC3 excise, most histology was benign but there were five malignancies, giving a PPV for malignant histology of AC3 excise of $21.7 \%$ in this series. For one of the five malignant cases there was an initial FNA of AC3 excise, 
with a later FNA being reported as AC5; the first FNA result presumably reflects sampling error.

Most of the histology following AC4 and AC5 FNAs was malignant, with an overall PPV of $90.3 \%$ (AC4, 68.2\%; AC5, 99.1\%). Fourteen AC4 reports were followed by benign histology. Four of these biopsies were cores showing normal breast tissue (three) and micropapillary hyperplasia (one) but, as described above, cores can give false negative results and further follow up is required. Ten biopsies were open/excision: two showed normal breast tissue, the remainder showed various benign changes including focal lactation (two), intraduct papilloma, sclerosing papilloma, complex sclerosing lesion, fibrocystic change with fibroadenomatoid hyperplasia, and two fibroadenomas - one with atypical lobular hyperplasia. The three AC4 reports not followed by histology related to one recurrence in a young patient under oncological care, one clinical malignancy in an elderly woman, and one ongoing case.

Forty four AC5 FNAs were not followed by histology. These were mostly from elderly patients due to undergo medical treatment, although a few cases were recurrences in younger patients. One AC5 report was accompanied by benign histology but this was a core biopsy only, showing atypical ductal hyperplasia that could possibly represent DCIS; the patient was an elderly woman with clinically obvious bilateral malignancy and primary medical treatment has been given with no intention of further biopsy.

DPAS STAINING

The slides stained with DPAS produced a variety of intracytoplasmic and extracellular staining patterns. Strict criteria were applied when classifying intracellular staining as positive, as described above. The size of such intracellular deposits was variable. The largest were some-

\section{A}
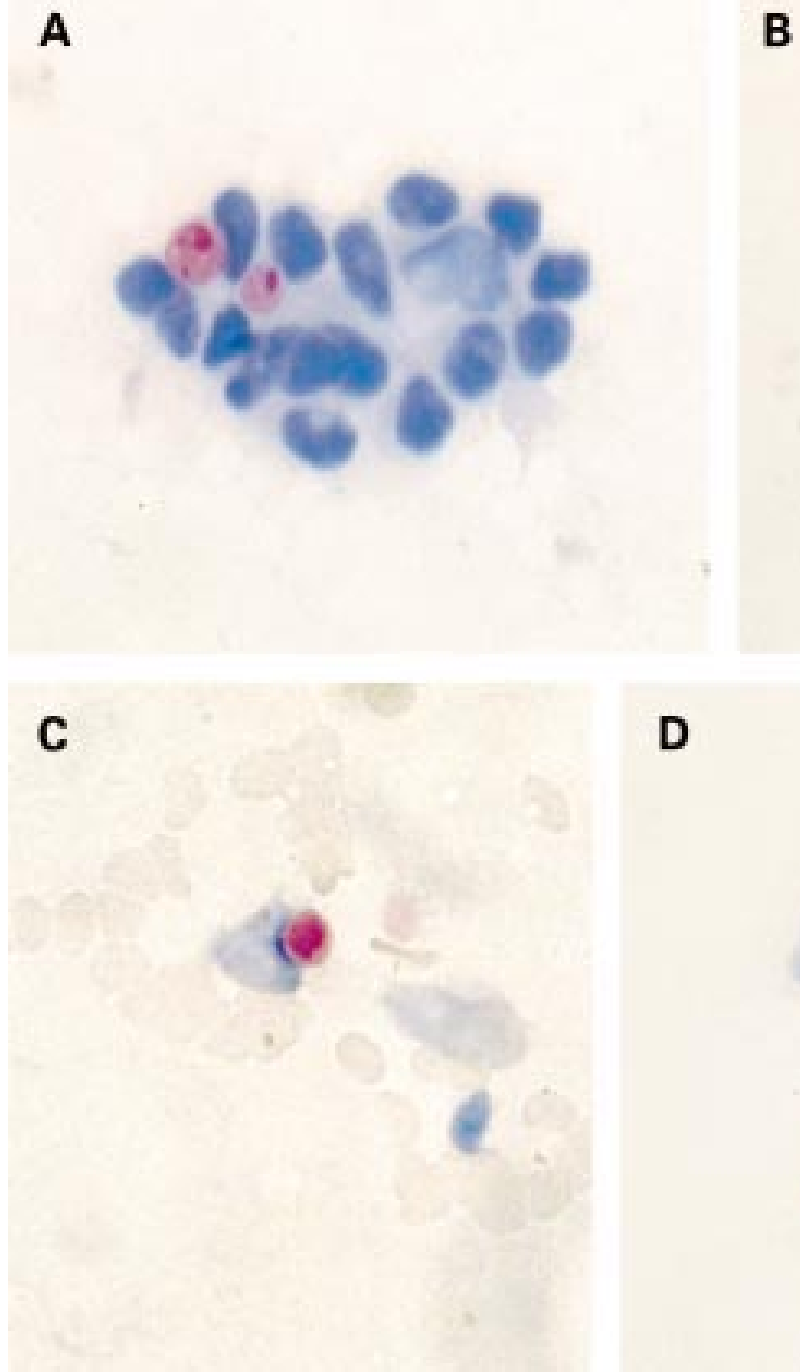

D

Figure 1 Acceptable diastase resistant periodic acid Schiff (DPAS) positivity. (A) A group of malignant epithelial cells two of which contain intracytoplasmic magenta body-like DPAS positivity with indentation of the adjacent nuclear membrane (DPAS stain, AC5); (B) and (C) dissociated malignant epithelial cells showing structured intracytoplasmic DPAS positivity with indentation of the adjacent nuclear membrane (DPAS stain, AC5); (D) a group of malignant epithelial cells containing intracytoplasmic DPAS positivity that sometimes lacks internal structure but does produce nuclear membrane indentation (DPAS stain, AC5). 
Table 2 Diastase resistant periodic acid Schiff (DPAS) scoring within the different aspiration cytology $(A C)$ grades

\begin{tabular}{|c|c|c|c|c|c|}
\hline \multirow{2}{*}{$\begin{array}{l}A C \text { grade as finally } \\
\text { reported }\end{array}$} & \multirow[b]{2}{*}{ DPAS score } & \multirow{2}{*}{$\begin{array}{l}\text { Number of } \\
\text { cases }\end{array}$} & \multicolumn{3}{|c|}{ Histology result } \\
\hline & & & No histology & Benign & Malignant \\
\hline \multirow[t]{5}{*}{$\mathrm{AC} 2$} & - & 123 & 113 & 9 & 1 \\
\hline & \pm & 7 & 7 & 0 & 0 \\
\hline & + & 7 & 6 & 1 & 0 \\
\hline & ++ & 0 & 0 & 0 & 0 \\
\hline & +++ & 0 & 0 & 0 & 0 \\
\hline \multirow[t]{5}{*}{ AC3 minor atypia } & - & 38 & 30 & 7 & 1 \\
\hline & \pm & 3 & 3 & 0 & 0 \\
\hline & + & 8 & 7 & 0 & 1 \\
\hline & ++ & 1 & 1 & 0 & 0 \\
\hline & +++ & 0 & 0 & 0 & 0 \\
\hline \multirow[t]{5}{*}{ AC3 excise } & - & 16 & 1 & 11 & 4 \\
\hline & \pm & 3 & 0 & 2 & 1 \\
\hline & + & 6 & 2 & 4 & 0 \\
\hline & ++ & 1 & 0 & 1 & 0 \\
\hline & +++ & 0 & 0 & 0 & 0 \\
\hline \multirow[t]{5}{*}{ AC4 } & - & 15 & 0 & 3 & 12 \\
\hline & \pm & 2 & 0 & 0 & 2 \\
\hline & + & 22 & 3 & 9 & 10 \\
\hline & ++ & 8 & 0 & 2 & 6 \\
\hline & +++ & 0 & 0 & 0 & 0 \\
\hline \multirow[t]{5}{*}{ AC5 } & - & 25 & 4 & 0 & 20 \\
\hline & \pm & 10 & 3 & 0 & 7 \\
\hline & + & 44 & 16 & 1 & 27 \\
\hline & ++ & 56 & 14 & 0 & 42 \\
\hline & +++ & 20 & 6 & 0 & 14 \\
\hline
\end{tabular}

times obvious lumina or vacuoles with the typical internal structure of a magenta body, having a dark outer ring with an inner clearer zone and a central pink dot (fig $1 \mathrm{~A}-\mathrm{C}$ ). The smaller examples were harder to find and assess, and were usually only accepted when producing a small indentation of the adjacent nuclear membrane, although to a much lesser degree than seen in a true signet ring cell. These smaller deposits usually lacked any internal structure and stained uniformly pink (fig 1D). Intracytoplasmic staining meeting these strict criteria was scored as positive (table 2).

Alternative patterns of intracytoplasmic staining were present but were not accepted as true positivity. These included residual glycogen granules not digested by the diastase, which were seen as multiple pink granules within the cytoplasm (fig 2A); these were especially obvious in apocrine cells of fibrocystic change. In addition, fluffy indistinct pink staining was seen in some cell cytoplasm but this was disregarded.

Extracellular DPAS staining included luminal material within groups of benign cells, especially in aspirates with features suggestive of a fibroadenoma (fig 2B). Care had to be exercised with smaller deposits that can mimic intracytoplasmic labelling but lack the nuclear indentation or internal structure. Cases of mucinous carcinoma contained extracellular pools of DPAS positive material, often traversed by capillaries.

\section{CORRELATION OF DPAS STAINING WITH}

HISTOLOGY

There was an association between acceptable DPAS positivity $(+,++,+++)$ on FNA and subsequent malignant histology (table 2). DPAS staining of ++ and +++ was seen almost exclusively in malignant cases. The overall PPV for malignant histology of a positive DPAS score was $84.4 \%(70.6 \%$ for,$+ 94.1 \%$ for ++ , and $100 \%$ for +++$)$. However, when all cases with malignant histology were considered there was a full range of DPAS staining from negative to strongly positive, with $66.1 \%$ scoring,+++ or +++ , and $41.9 \%$ scoring ++ or +++ .

Most of the benign aspirates (AC2 and AC3) were DPAS negative. Only a few benign cases contained occasional equivocal $( \pm)$ or positive $(+)$ cells, and these were usually present within a flat sheet of non-atypical epithelial cells with overlying sentinel nuclei. The only example of ++ staining in an AC3 minor was in a patient receiving drugs to stimulate ovulation. The only AC3 excise case with ++ staining contained atypical epithelial and stromal cells; an open biopsy showed a fibroadenoma with mucoid stroma.

Many of the AC4 cases and most of the AC5 cases were DPAS positive, with AC5 FNAs containing the highest proportion of ++ and +++ staining. Two AC4s had DPAS scores of ++ and benign histology: both were excision biopsies, one showing focal lactational change,
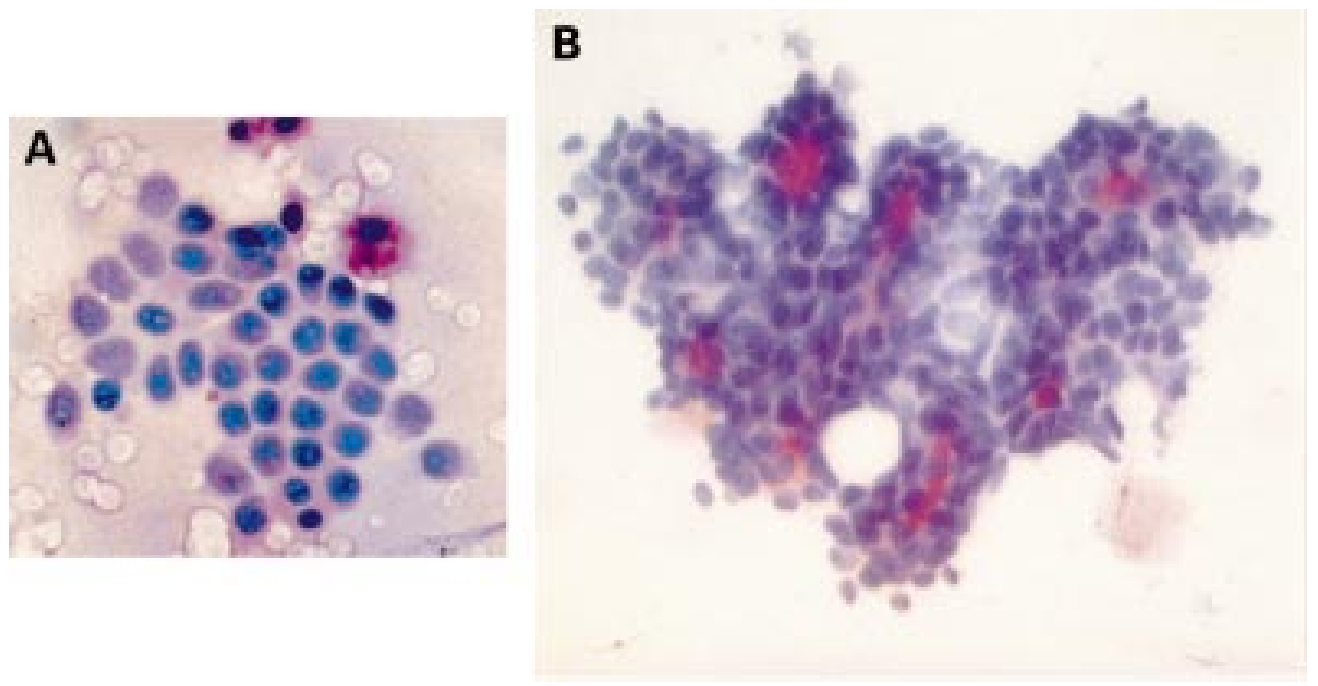

Figure 2 Unacceptable diastase resistant periodic acid Schiff (DPAS) positivity. (A) A sheet of benign epithelial cells at the edge of which is a cell containing intracytoplasmic glycogen granules staining positively for DPAS (DPAS stain, AC2); (B) a sheet of benign epithelial cells demonstrating branching luminal DPAS positivity (DPAS stain, AC2). 
Table 3 Correlation of diastase resistant periodic acid Schiff (DPAS) scores with subsequent malignant histology

\begin{tabular}{|c|c|c|c|c|c|c|c|c|c|c|c|}
\hline \multirow[b]{2}{*}{$\begin{array}{l}\text { DPAS } \\
\text { score }\end{array}$} & \multirow{2}{*}{$\begin{array}{l}\text { Number of cases } \\
\text { with malignant } \\
\text { histology }\end{array}$} & \multicolumn{2}{|c|}{ In situ only } & \multicolumn{8}{|c|}{ Invasive carcinoma types and grades } \\
\hline & & DCIS & LCIS & $\begin{array}{l}\text { Ductal } \\
\text { grade } 1\end{array}$ & $\begin{array}{l}\text { Ductal } \\
\text { grade } 2\end{array}$ & $\begin{array}{l}\text { Ductal } \\
\text { grade } 3\end{array}$ & $\begin{array}{l}\text { Ductal } \\
\text { ?grade }\end{array}$ & Lobular & $\begin{array}{l}\text { Ductal/ } \\
\text { lobular }\end{array}$ & Tubular & Other \\
\hline - & 38 & 7 & 1 & 2 & 13 & 9 & 4 & - & - & 2 & - \\
\hline \pm & 10 & - & - & 1 & 4 & 4 & - & - & - & 1 & - \\
\hline+ & 36 & 6 & - & 12 & 7 & 8 & 2 & - & - & 1 & - \\
\hline++ & 48 & 6 & - & 11 & 15 & 9 & - & 1 & 1 & 1 & $\begin{array}{l}1 \mathrm{med} \\
1 \mathrm{muc} \\
2 \mathrm{LN} \\
\text { met }\end{array}$ \\
\hline+++ & 14 & - & - & - & 4 & 4 & 1 & 3 & 1 & - & $1 \mathrm{muc}$ \\
\hline Total & 144 & 19 & 1 & 26 & 43 & 34 & 7 & 4 & 2 & 5 & 5 \\
\hline
\end{tabular}

DCIS, ductal carcinoma in situ; LCIS, lobular carcinoma in situ; LN met, lymph node metastasis from breast carcinoma; med, medullary carcinoma; muc, mucinous/colloid carcinoma.

and the other a duct papilloma. DPAS positivity in the malignant cases was characteristically seen within atypical epithelial cells that were either dissociated or in poorly cohesive groups. The stained deposit often produced nuclear indentation and/or had an obvious internal structure, sometimes with a central dot. Intracytoplasmic lumina had been evident on DQ or MGG staining in some of these.

Six cases were upgraded to AC4 on a positive DPAS result, two from an immediate report of AC3 minor atypia and four from AC3 excise. In one case there was no histology. In five cases the histology was benign: one core biopsy showed normal breast tissue; four excision biopsies showed one fibrocystic change with fibroadenomatoid hyperplasia, two fibroadenomas (one with atypical lobular hyperplasia), and a sclerosing papilloma. Each had a DPAS score of + . Review of the DPAS staining showed that the positive cells were usually within sheets of relatively bland epithelium with overlying sentinel nuclei, and the positivity was mostly seen as small round globules with only subtle nuclear indentation. The case of the sclerosing papilloma showed DPAS positivity in small atypical cells in groups but with convincing nuclear indentation.

Two cases were upgraded to AC5 from an immediate report of AC4 on the basis of a DPAS score of + , and each was a grade 1 invasive ductal carcinoma on histology. On review, the DPAS positive globules were within atypical cells (dissociated or in loose groups), had internal structure, including central dots, and indented the nuclear membrane.

Table 3 shows DPAS scores correlated to tumour types for the cases with malignant histology. As expected, most malignancies were invasive ductal carcinoma (carcinoma of no special type) although small numbers of lobular, tubular, medullary, and mucinous types were included in this series.

\section{Discussion}

FNA cytology is invaluable in the preoperative investigation of breast lesions and in skilled hands is highly accurate. In some centres there is an unfortunate trend towards the replacement of FNA with needle core biopsies for histological evaluation, suggesting some local loss of confidence in the accuracy of FNA diagnosis. We believe that both modalities have important roles and that the most accurate preoperative diagnoses will result from using them side by side in selected cases. Any technique that further improves the diagnostic accuracy of breast FNA is therefore of value to both screening and symptomatic breast services.

The cytological diagnosis of breast lesions has been well described in standard texts, ${ }^{2-4}$ and is familiar to all of us routinely involved in breast FNA evaluation. Most adequate specimens are straightforward, being either benign or malignant. Benign samples show sheets of uniform epithelial cells often with overlying sentinel nuclei (myoepithelial cells) and with bare sentinel nuclei in the background. Malignant aspirates are characterised by epithelial cell dissociation, nuclear enlargement, and nuclear atypia, often with cytoplasmic vacuolation. However, there are a small number of problematical specimens. Such cases usually have varying degrees of epithelial cell atypia and/or dissociation, with interpretation often complicated by scantiness of the specimen, small sized cells, or an admixture of the atypical cells with benign elements. These cases tend to fall into the AC3 and AC4 categories, which are less helpful to the clinicians than a diagnostic report.

In our study, the PPVs for malignancy were $21.7 \%$ for AC3 excise, $68.2 \%$ for AC4, and 99.1\% for AC5. The PPV for AC3 excise is broadly in line with our usual results. The PPVs for AC4 and AC5 are below our usual standard of over $90 \%$ and $100 \%$, respectively. The large shortfall for AC4 probably reflects in part the inclusion of only those cases with sufficient cells to perform DPAS staining on an entire slide, namely 47 cases, with exclusion of the remaining $22 \mathrm{AC} 4 \mathrm{~s}$ of lower cellularity that were reported during this time period (histology on 15: one benign, one equivocal, and 13 malignant). The inappropriate upgrading to AC4 of six cases on the basis of DPAS staining during our study also distorts the PPV results. The shortfall in the PPV for AC5 reflects the one case with an inconclusive core biopsy but clinical malignancy.

DPAS positive intracytoplasmic lumina have been demonstrated in histological sections in all types of breast carcinoma, but particularly lobular carcinoma, ${ }^{5}$ producing the so called target cells. Ultrastructural assessment has revealed condensation of cytoplasm around the lumina, producing a thick wall, with internal 
microvilli and central electron dense material, the latter perhaps corresponding to the central dot of the magenta bodies seen in the cytology of breast carcinoma. ${ }^{56}$ Intracytoplasmic lumina on FNA have been described as characteristic of lobular carcinoma. ${ }^{1-4} 7$ Signet ring cells have been found in both lobular carcinoma and ductal carcinoma, and cannot be relied on to predict tumour type. ${ }^{8-11}$ Intracellular secretory vacuoles have also been reported in cytology specimens from less common breast carcinoma types, including high grade mucoepidermoid carcinoma, ${ }^{12}$ secretory carcinoma, ${ }^{6}$ spindle cell argyrophilic mucin producing carcinoma, ${ }^{13}$ signet ring cell carcinoma, ${ }^{14}$ and mucinous carcinoma. ${ }^{15}$ Although a few authors describe DPAS positivity in vacuolated cells on histological sections from these tumour variants, ${ }^{5} 91016$ none mention using mucin stains on the cytology preparations to assist in diagnosis.

We have long used DPAS staining to detect intracellular mucin in breast FNAs, mostly to assist in making a definite diagnosis of malignancy (AC5) in an otherwise suspicious case (AC4). DPAS staining is a simple and quick technique that can be performed on either unstained or destained DQ or MGG slides, and can easily be incorporated into routine cytology laboratory staining. The results presented here show a strong correlation between cytological intracellular DPAS positivity and subsequent malignant histology.

The DPAS result must be interpreted in conjunction with the routine cytological appearances. Strict criteria are essential for the correct interpretation of positive staining and experience with many previous aspirates is valuable. DPAS positivity is most reliable when seen in more than just a few cells (scores of ++ or +++ ) and should be present within atypical epithelial cells, which are usually dissociated or in loose groups. The DPAS positive globules may vary in size but should clearly indent the nuclear membrane. Internal structure within the globule, such as a central magenta body-like dot, is reassuring. In the series presented here, we reliably upgraded two FNAs from AC4 to AC5 based on such DPAS positivity.

DPAS staining is less reliable as an indicator of malignancy when equivocal or seen in only occasional cells (scores of \pm or + ), when present within sheets of otherwise bland epithelium with overlying sentinel nuclei, and when the nuclear membrane is not indented by the globule. Over enthusiastic interpretation of such staining may lead to overcalling of the FNA as we have demonstrated. In hindsight, attention to this would have avoided the upgrading to AC4 of five of the six cases. Sethi and colleagues $^{9}$ and Sturgis and colleagues ${ }^{10}$ also cautioned against overinterpretation of vacuolated cells within sheets of epithelial cells and considered intracytoplasmic lumina to be of most value when seen in dissociated atypical cells, although they did not describe DPAS staining. Such atypical cells are sometimes scanty and/or small in size, and it is in these situations that appropriate DPAS positivity is of particular value.

In conclusion, FNA is a widely used, rapid, and accurate technique for the preoperative assessment of breast lesions. Intracellular DPAS positivity within atypical cells in these aspirates predicts malignancy and may assist in upgrading from a suspicious to a diagnostic result.

We are indebted to Mrs J Moore, Mrs L VandeMast, Mr A Bright, Mrs S Carter, and Miss S Graysmark for assistance with Bright, $M$.
this study.

1 Nicholson S, Sainsbury JRC, Wadehra V, et al. Use of fine needle aspiration cytology with immediate reporting in the needle aspiration cytology with immediate reporting in

2 Coghill SB. Normal breast cytology and breast screening. Inflammatory conditions and benign breast lesions. Malignant breast tumours. In: Gray W, ed. Diagnostic cytopatholggy. Edinburgh: Churchill Livingstone, 1995:225-98.

3 Silverman JF. Breast. In: Bibbo M, ed. Comprehensive cytology, 2nd ed. Philadelphia: WB Saunders, 1997:73180.

4 Orell SR, Lindholm K. Breast. In: Orell SR, Sterrett GF, Walters MN-I, et al, eds. Manual and atlas of fine needle aspiration cytology, 2nd ed. Edinburgh: Churchill Livingstone, 1992:129-69.

5 Battifora H. Intracytoplasmic lumina in breast carcinoma. Arch Pathol 1975;99:614-17.

6 Pohar-Marinšek Z, Golouh R. Secretory breast carcinoma in a man diagnosed by fine needle aspiration biopsy. Acta Cytol 1994;38:446-50.

7 Salhany KE, Page DL. Fine-needle aspiration of mammary lobular carcinoma in situ and atypical lobular hyperplasia. Am 7 Clin Pathol 1989;92:22-6.

8 Greeley CF, Frost AR. Cytologic features of ductal and lobular carcinoma in fine needle aspirates of the breast.
lobeley Acta Cytol 1997;41:333-40.

9 Sethi S, Cajulis RS, Gokaslan ST, et al. Diagnostic significance of signet ring cells in fine-needle aspirates of the breast. Diagn Cytopathol 1997;16:117-21

10 Sturgis CD, Sethi S, Cajulis RS, et al. Diagnostic significance of "benign pairs" and signet ring cells in fine needle aspirates (FNAs) of the breast. Cytopathology 1998; 9:308-19.

11 Frost AR, Terahata S, Yeh IT, et al. The significance of signet ring cells in infiltrating lobular carcinoma of the breast. Arch Pathol Lab Med 1995;119:64-8.

12 Pettinato G, Insabato L, de Chiara A, et al. High grade mucoepidermoid carcinoma of the breast-fine needle aspiration cytology and clinicopathologic study of a case. Acta Cytol 1989;33:195-200.

13 Burgan AR, Frierson HF, Fechner RE. Fine-needle aspiration cytology of spindle-cell argyrophilic mucinaspiration cytology of spindle-cell argyrophilic mucin-
producing carcinoma of the breast. Diagn Cytopathol 1996; producing car

14 Kamiya M, Mizuguchi K, Yoshimoto M, et al. Cytologic diagnosis of signet-ring cell carcinoma of the breast. Acta Cytol 1998;42:650-6.

5 Dawson AE, Mulford DK. Fine needle aspiration of mucinous (colloid) breast carcinoma-nuclear grading and mammographic and cytologic findings. Acta Cytol 1998;42: 668-72.

16 Gad A, Azzopardi G. Lobular carcinoma of the breast: a special variant of mucin-secreting carcinoma. 7 Clin Pathol 1975;28:711-16. 